\title{
Rigidity in Cinema Seen From the Front Row, Side Aisle
}

\author{
James E. Cutting \\ Cornell University
}

\begin{abstract}
Pictures and cinema seen at a slant present the cotics of virtual objects that are distorted and inconsistent with their real counterparts. In particular, it should not be possible for moving objects on slanted film and television screens to be seen as rigid, at least according to rules of linear perspective. Previous approaches to this problem have suggested that some process (perhaps cognitive) rectifies the optics of objects in slanted pictures to derive true shape and preserve shape constancy. The means for this rectification is usually thought to be based on recovery of true screen slant. In three experiments I show that this account is unnecessary and insufficient to explain the perception of rotating, rectangular objects in slanted cinema. I present data in favor of an alternate view, one in which the information is sufficient for perceivers to determine rigidity in an object on slanted screens, at least for parallel projections. In the human visual system, local measurements of objects are apparently made according to projective geometry; in those measurements, small amounts of certain distortions in projection are tolerated. Stimuli that appear nonrigid are ones that violate certain local principles, known as Perkins's laws, of projections of rectangular solids.
\end{abstract}

Eye position is not fixed when one looks at a photograph or painting. A puzzle arises from this fact: Linear perspective, the means of representation in photos and in many works of art, is mathematically correct for only one viewpoint. I call this point the composition point; it is the point from which a photograph was taken (the perpendicular distance from the center of the photograph derived by multiplying the focal length of the lens by the degree of enlargement) or the point from which a linear perspective picture was composed according to the rules of Giambattista Alberti (e.g., White, 1957).

Fortunately, picture viewing is not limited to the composition point. In fact, a large number of positions in front of a picture will serve as reasonable viewpoints, will preserve object identity, and will allow layout within the picture to appear relatively normal. Preservation of phenomenal identity and phenomenal shape of objects in slanted pictures is fortunate, for without them the utility of pictures would be vanishingly small. Nevertheless, both are unpredicted by any theoretical application of linear perspective, and the second of these-the relatively unnoticed distortions in slanted pictures and cinema-is the focus of this article.

The puzzle of unnoticed distortions in slanted images was first addressed by La Gournerie in 1859 (Pirenne, 1970);' hence

This research was supported in part by National Institutes of Health Grant MH37467 and by the graciousness of the Department of Psychology of the University of Arizona during my sabbatical leave from Cornell. The data of Experiment 1 were first reported at the 26th meeting of the Psychonomic Society, Boston, November 1985.

I thank Michael Kubovy for retriggering my interests in slanted cinema, Ennio Mingolla for discussions about its optics, and Joseph Lappin and two anonymous reviewers for suggestions about improvements in this article.

Correspondence concerning this article should be addressed to James E. Cutting Department of Psychology, Uris Hall, Cornell University, Ithaca, New York 14853-7601.
I call it La Gournerie's paradox (Cutting, 1986a, 1986b). Kubovy (1986) called it the robustness of perspective. Slanted pictures have since received attention from Gibson (1947), Perkins (1973), Hochberg (1978a), Farber and Rosinski (1978), Rosinski and Farber (1980), Rosinski, Mulholland, Degelman, and Farber (1980), and Lumsden (1980), but none of these authors followed the strategy that I present here. La Gournerie's paradox occurs in two forms: The first concerns viewing pictures either nearer or farther than the composition point but along the line extended between that point and (usually) the center of the picture; the second, and by far the more interesting and complex, concerns viewing pictures from the side at any distance. Both are discussed below, but to do them justice, one must first discuss the shape of virtual space.

\section{La Gournerie's Paradox and the Reconstruction of Virtual Space}

To consider distortions of images seen from noncomposition points, one must perform, as La Gournerie did, some reconstructive geometry, rebuilding the virtual space in which the objects are depicted. The premise for doing so is the reversal of the image registration process: Rather than following the ambient light of the real world "passing through" the picture plane and bathing the eye, the paths of reconstruction go from the eye, through the fixed points on the picture surface, and into depth behind the picture. This reversal is not intended as a theory of picture perception, but it is, I suggest, the way to begin to understand the layout of objects in virtual space.

Before proceeding with such reconstructions, however, there is an important caveat to consider. It is often noted that the

\footnotetext{
' Discussions of distortion in slanted pictures began eartier with Leonardo, Cousin, and Lomazzo in the 16th century (Frangenberg, 1986). Nonetheless, it was La Goumerie who fostered the idea of reconstructing distortions in slanted pictures.
} 
three-dimensional layout behind any static, two-dimensional image is ambiguous (e.g., Hochberg, 1978b, p. 55; Rock, 1983, p. 22). This fact appears to make the whole enterprise of reconstruction untenable. My counterclaim is that this notion would be misapplied in most computational approaches to vision, of which $L a$ Goumerie's can be taken as a very early example. That is, ambiguity of three-dimensional form represented in two-dimensional images is not true under certain constraints, or assumptions. Moreover, it is the discovery of possible assumptions that yield unambiguous solutions that is central to the enterprise. ${ }^{2}$ Among them are that (a) the observation point is a nontrivial distance above a ground plane, (b) the ground plane is cpaque, and (c) the environment is composed of opaque objects lying on that plane. If one adds the assumption that all principal faces of portrayed objects have right-angled corners, then the task of reconstruction becomes computationally easy (Greene, 1983; Kubovy, 1986). Right corners have been assumed in many approaches to object perception (e.g., Barnard, 1985; Guzman, 1969; Perkins, 1972, 1982; Shepard, 1981), and aithough this assumption is generally untenable for natural and many artificial objects, I use it here. My rationale is straightforward: Because I am studying images seen from noncomposition points, it is helpful to use rectilinear objects with orthogonal surfaces because they tractably reveal the true composition point in linear perspective and thus would best serve slanted picture perception.

Reconstruction proceeds from the relative positions of observer and picture. From the composition point, a viewer may change his or her position in front of a picture in two general ways: (a) to and fro along the $z$ axis, orthogonal to the picture plane, and (b) laterally along the $x$ or $y$ axis, parallel to it. Both observer movements generate affine transformations in depth, the $x z$ plane of virtual space. (Affine transformations are those in which parallel lines remain parallel after transformation but where angles between intersecting lines are generally changed.) Only the second, or lateral, observer movement generates perspective transformations, in which parallel lines no longer remain parallel. Both are indicated in Figure 1. In the left panels, four pillars are projected onto the image plane as might be seen in a large poster. The upper panel shows the spatial layout in the horizontal $(x z)$ plane; the lower shows the projection itself. For all noncomposition viewpoints the locations of pillars in depth are determined by a simple ratio rule. The ratio is the distance from the composition point to an object $(D)$ divided by the distance from the composition point to the location on picture that the object is represented $(d)$. The particular ratio $D / d$ associated with every object part is preserved at all noncomposition points. Thus, knowing the position of the object on the picture surface, one need only continue the line from the noncomposition point, through the surface, to the appropriate depth in virtual space where the ratio is preserved.

When the observer moves closer to the image, as in the middle panels of Figure 1 , the projected pillars stay in the same physical locations in the photo. This means that the geometry in the virtual environment behind the image must change. Notice that the distance between front and back pairs of this four-pillared scene is compressed, a collapse of depth like that viewed through a telephoto lens. ${ }^{3}$ All changes in $z$-axis location of the observer create such compressions (or expansions) of the object
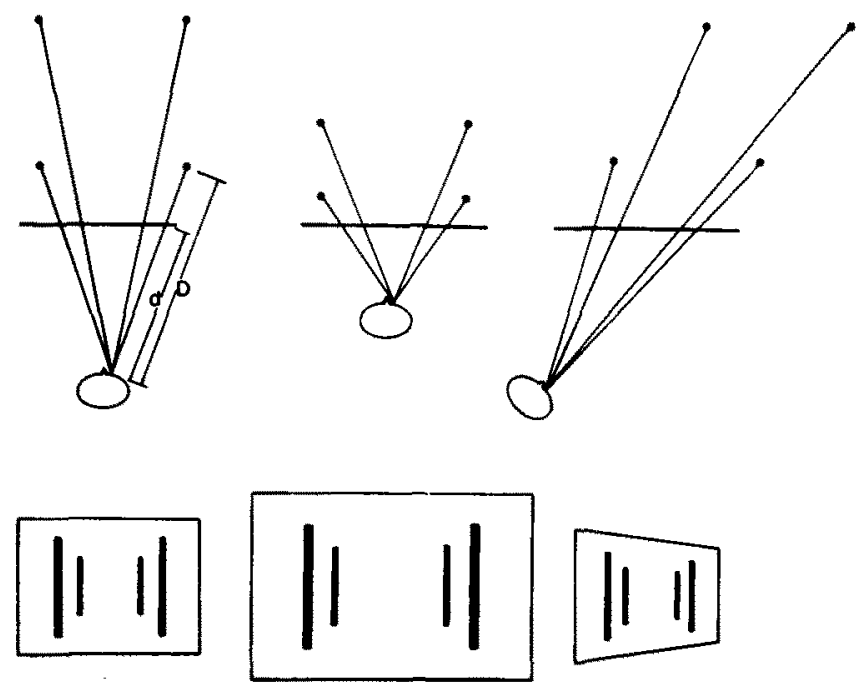

Figure 1. Reconstructive geometry and images. (Left panels show the nondistorted geometry of four pillars arranged in a square, the upper panel as if seen from above and the lower as a picture of them. Middle panels show the distortions of pictures seen close up; the upper panel demonstrates the compression of depth. Right panels show the distortions of slanted pictures; the upper panel demonstrates the affine shear of all $x z$ slices in depth, and the lower panel demonstrates the perspective deformation of all $x y$ stices in depth. $D$ is the distance from the composition point to a particular object part when the picture was composed, and $d$ the distance from the composition point to the corresponding point in the image. The ratio $D / d$ is constant for all reconstructions of each point. From "The shape and psychophysics of cinematic space" by James E. Cutting, 1986, Behavior Research Methods, Instruments, and Computers, 18, p. 553. Copyright by The Psychonomic Society. Adapted by permission.)

in virtual space. These virtual space changes are affine transformations; no perspective transformations occur with this type of change in observer viewpoint.

When an observer moves to the side, as seen in the right panels of Figure 1, the pillars in virtual space must shif over. Moreover, they do so by differing amounts. Such shifts are due to affine shear or all horizontal planes in the virtual space. These generate an oft-noted effect in portraiture: If the subject of the portrait appears to look out at the observer when he or she is in front of the painting, it will also appear to follow the observer around the room (e.g., Anstis, Mayhew, \& Morley, 1969; Pirenne, 1970; Wallach, 1976). All viewpoints of a picture yield additive combinations of these two affine effects: compression or expansion of the $z$ axis, and shear of that axis against the picture (frontoparallel) plane.

Changing viewer position to the side, however, also adds a perspective transformation, as shown in the lower right panel.

\footnotetext{
${ }^{2}$ To be sure, reconstruction of metrical information, such as absolute size of objects in virtual space, is not possible by purely reconstructive techniques.

${ }^{3}$ Approach and recession by the observer also changes the optical proportions of the vertical height and horizontal width of the rectangular poster. (See Cutting, 1986a, pp. 163-167.)
} 

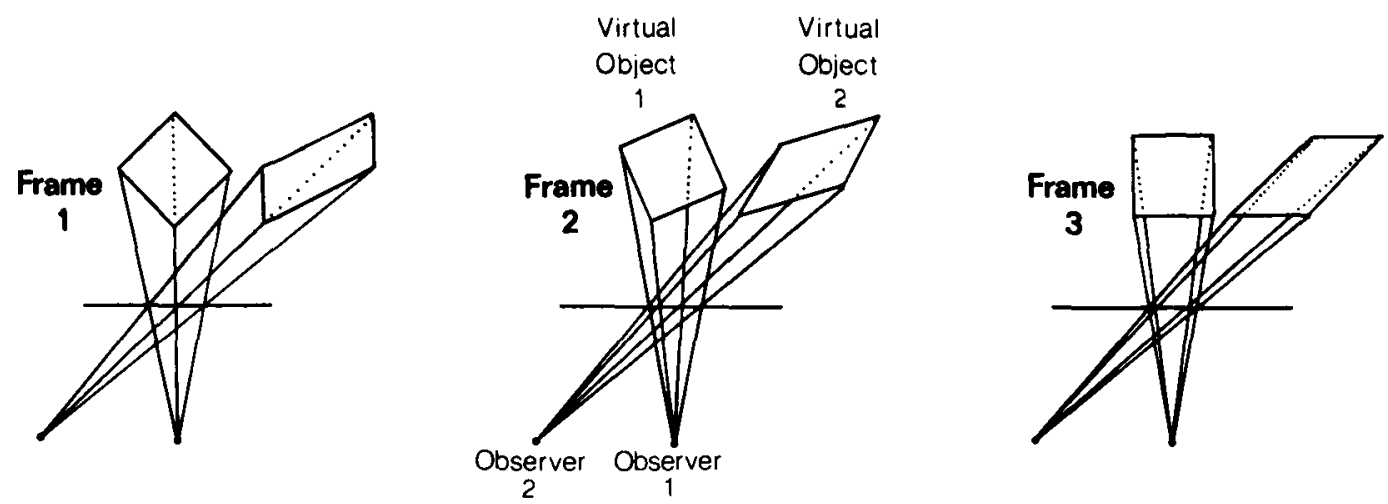

Figure 2. Two virtual objects reconstructed behind the image plane, one for an observer at the composition point and the other for an observer well of to the side. (In reconstruction, an affine transformation is assumed in all $x z$ planar slices through virtual space; distance is kept between frontal plane slices parallel to the picture plane but with considerable affine shear. Across the three panels, the virtual objects rotate 45. Virtual Object 1 for Observer 1 is rigid in Euclidean space; Virtual Object 2 for Observer 2 undergoes plastic deformations. Here only the affine deformations are shown; perspective deformations would be superimposed on all $x y$ slices through virtual space. From "The shape and psychophysics of cinematic space" by James E. Cutting, 1986, Behavior Research Methods, Instruments, and Computers, 18, p. 554. Copyright by The Psychonomic Society. Reprinted by permission.)

Here pillars near the left edge of the image are relatively larger than their counterparts near the right edge. More specifically, all $x y$ slices in depth, parallel to the picture plane, have undergone the same perspective transformation.

\section{The Central Problem in the Perception of Cinema}

Thus far I have discussed La Gournerie's paradox only for static images. However, the paradox is compounded in cinema (Hochberg \& Brooks, 1978), a term used for both television and film. In fact, the paradox is sufficiently profound that I consider it the most important problem in cinema perception. ${ }^{4}$ The problem manifests itself for all viewers. The composition point in cinema is at the location of the projector for film shot and projected with the same power lens. Because no viewer can sit in this location, La Gournerie's paradox, to greater and lesser degrees, is pertinent to all cinema viewers. But, what is more striking, one can sit near the screen and well to the side-viewing a large trapezoidal screen-and a rotating object in the film will not appear to deform. In such a location, the assumption of right corners of the virtual object must be false under any and all of its reconstructions in virtual space. More generally, from all noncomposition points, any reconstruction of the shape of the virtual object would show that it continually undergoes plastic deformation. Figure 2 illustrates an example. Given the perceived rigidity but reconstructive plasticity of the object, the visual system must compensate for, ignore, or not register such stimulus changes.

\section{Three Explanations for La Gournerie's Paradox}

Consider three candidate explanations for La Gournerie's paradox, ones that I believe exhaust the possibilities. It may be that (1) the distortions of virtual space are detransformed by some cognitive process, rectifying the original relations as com- posed in the picture. On the other hand, the visual system might simply use the information in the display as it survives the perspective and affine transformations. This second idea divides two ways: (2) The survival of the visual information may be absolute, in which case the information is invariant under transformation, or (3) the transformation of the information may be too small to be discerned by the visual system. These three explanations, in a given perceptual situation, need not be mutually exclusive, but it is useful to try to separate them.

Approach 1: Euclidean rectification. Most explanations for the perception of pictures at a slant are in sympathy with the general perceptual theory of Helmholtz. Pirenne (1970), for example, suggested that "an unconscious intuitive process of psychological compensation takes place, which restores the correct view when the picture is looked at from the wrong position" (p. 99). Pirenne's unconscious inference and similar schemes proposed by Greene (1983) and Kubovy (1986) appear to unpack the transformations through some process akin to mental rotation (Shepard \& Cooper, 1982). I call this notion the Euclidean rectification hypothesis. According to it, the viewer detransforms the distortions in pictured space, in essence, so that things may be seen properly. Although my concern is not with static photographs, the same theoretical perspective might hold for viewing film from the front row, side aisle. Any static frame or collection of frames might be used to determine the proper station point; in fact, given many related but nonidentical frames, the task of rectification might be computationally easier than from a single static frame.

I find two problems with the Euclidean rectification view,

\footnotetext{
- Many other problems could be noted-for example, how cuts work (Hochberg \& Brooks, 1978). I claim that La Gournerie's paradox is prior because without it the efficacy of film and television would be so limited that cuts might never have been invented.
} 
whether for photos or film. First, it requires the observer to recover the absolute slant of a particular part, typically the center, of the image. If the viewing angle is, say, $75^{\circ}$, the viewer must perform the compensatory work to "change" the image so that it is $90^{\circ}$, orthogonal to the picture plane. Unfortunately, the empirical problem with such recovery is that observers are not very accurate in determining the slant of a highly textured, flat surface (Braunstein \& Payne, 1969; Epstein, Bontrager, \& Park, 1962; Perrone, 1984), much less a glossy or matte sheet of paper or a glass-bead projection surface. Results show that slant is typically underestimated, making rectification incomplete. Second, Euclidean rectification entails something like "double perception"; that is, the observer first looks at the image and then rectifies it, as if to then look at it again. Such a process seems redundant, computationally cumbersome, and more difficult than normal picture perception. However direct picture perception might be, ${ }^{5}$ it seems parsimonious to have slanted and unslanted picture perception proceed in the same way.

Approach 2: Invariance under transformation. A second resolution to La Gournerie's paradox that seems possible, particularly for cinema, concerns invariance under transformation. Possible transformations are two: the slanted screen yielding the affine and perspective distortions noted previously and object motion. I call this notion the invariance hypothesis: First, there may be mathematical invariants that are preserved in cinematic images seen from the side; second, the visual system may use them. Elsewhere, I have promoted this idea (Cutting, 1986a, 1986b), using the example of the invariant cross ratio and how it is preserved and used for perception of planar objects on slanted screens.

Unfortunately, cross ratios are limited in scope. Their measurement is confined to four collinear elements (or coplanar parallel lines) as projected along one dimension. Thus, whereas cross-ratio invariance exemplifies an invariance account of La Gournerie's paradox, it is hardly general support. Moreover, I know of no more general invariant that might survive slanted screens, so rather than search for other mathematical entities, like the cross ratio, I will not discuss this possible explanation of La Gournerie's paradox. Instead, I turn to a third notion.

Approach 3: Indiscriminable distortions. It is often said that a human perceiver is something of a geometer (Olson, 1974; Perkins, 1982). A third explanation for La Gournerie's paradox in cinema, then, concerns a nonstandard idea about what kind of geometer a person might be. My claim is, in essence, that the visual system is a fuzzy geometer (Cutting, 1986a, chap. 4): Small, local deviations from Euclidean geometry and projections of it are simply not noticed in the perception of slanted photographs and cinema. I call this idea the indiscriminability hypothesis. It reduces to the idea that the visual system ignores certain affine and perspective transformations of virtual space because it never picks them up in the first place. ${ }^{6}$

Before discussing the technique that I use to separate the first and third approaches, let me briefly discuss how they would fit into the field of machine vision.

Structure from motion. Certain algorithms from machine vision research (e.g., Bennett \& Hoffman, 1985; Ullman, 1979, 1983) compute the rigid three-dimensional layout of various numbers of moving points from several static projections or from one static projection with vector paths associated with each point. The shape of a rigid configuration results from successful computations. This framework could be used equally well for the Euclidean rectification hypothesis and for the indiscriminability hypothesis. In the first, an additional parameter of screen slant could be tested for and derived from additional frames or more precise information about vectors, and in the other, recovery could be incremental, on the basis of the degree to which the data (point locations) fit the predictions. Because no machine vision research that I know of has addressed La Gournerie's paradox, no algorithm to deal with it is fleshed out. Ullman's (1984) incremental approach comes closest.

If the indiscriminability (or invariance) account(s) can be shown tenable, then the Euclidean rectification account must first be rendered unnecessary. To do this, I explore a new methodology - that of a simulated projection screen.

\section{Simulated and Real Projection Surfaces}

To remove the grounds for Euclidean rectification, one must consider how rectification might occur. Pirenne (1970) and others have suggested three sources of surface information in an image that might be used-the edges of the screen, which provide a trapezoidal frame of reference for the image; surface information such as the shape of specularities, bright spots showing the locations of room illumination and hence the relation of the screen to them; and binocular disparities, which grade uniformly across the slanted surface. I have already argued that these may not provide suitably rich information for the perceiver, but because I am not interested in them, I must remove them from my displays.

To eliminate framing and binocular cues from the perception of slanted cinema, one could have participants view stimuli through a monocular reduction screen. To eliminate specularity cues, one could polarize light sources. Indeed, Rosinski et al. (1980) did both. In general, however, there is a method less encumbering for the observer, although more encumbering for the computer programmer (Cutting, 1986b). As shown in the left panel of Figure 3, one can conceive of the front-row, sideaisle cinematic viewing situation as having a real projection screen (that in the cineauditorium) and an imaginary flat projection surface across which the stimulus optics are measured. Once it is conceived this way, one can reverse the two projection surfaces, as shown in the right panel, with the imaginary projection surface behind the real one. In the experiments discussed here, I employ this technique, which I call a double projection scheme. In this manner, the display scope always appears rectangular to the observer, removing frame-shape effects, surface re-

\footnotetext{
${ }^{5}$ Gibson (1979) regarded all picture perception as indirect. My claim is only that composition point and noncomposition point picture perception ought to have the same explanation, in terms of both process and information.

${ }^{6}$ It might seem somewhat tricky to separate this explanation from the previous one, but on the invariance account, I would argue, the visual system accurately registers the stimulus transformations but sifts through them to get at an invariant source of information. It is as if the transformation is measured and then filtered out, leaving the invariant as residual. On the indiscriminability account, on the other hand, the visual system simply does not register the transformations.
} 
flections, and graded disparities across the virtual screen surface, but the optical shapes of rotating stimuli are like those seen from the side.

This simulation yields transformations optically identical to those of a one-eyed observer moved to the side. First, there is a perspective transformation of the image screen and all parallel $x y$ slices of virtual space behind it, making object parts near one edge of the screen larger than those near the other. Second, there is the affine transformation of the stimulus in virtual space. As before, according to linear perspective, the intersections of adjacent sides of a rotating, rectangular solid would appear to hinge and the sides themselves deform. Moreover, the Euclidean rectification view should suggest that without trapezoidal framing effects, surface cues, and binocular disparities, these transformations should create the impression of nonrigidity in a rotating stimulus.

\section{General Method}

In all experiments viewers were presented with computer-generated, rotating, rectangular solids. Dimensions of all rigid solids, in normalized arbitrary units, were $1.3 \times 1.0 \times 1.0$ along the $x, y$, and $z$ axes, respectively. Stimuli subtended about $5^{*}$ of visual angle horizontally and vertically. Cubic objects were avoided so that projection lines of equal length could not be used as a source of information for rigidity judgments.

Stimuli were generated on a Hewlett-Packard (HP) 1000L Series minicomputer and shown on an HP 1350S display system with a P31 phosphor. Although this is a vector-plotting system without capabilities of shading, stimuli were not simply wire-frame constructions. Instead, objects were computed so that near sides occluded far ones; edge lines that should be hidden were removed from the stimulus. ${ }^{7}$ Sixty frames were calculated for each complete rotation, $6^{\circ}$ per frame. An experimental trial consisted of one object rotating three times. Each frame was shown for $57 \mathrm{~ms}$, yielding a trial duration of about $10.3 \mathrm{~s}$.

The design of each experiment was multifactor, with three factors carried across all experiments. These are discussed in detail below, but consider them in overview. First, the degree of perspective was varied; some rotating solids were seen as if they were quite close to the viewer
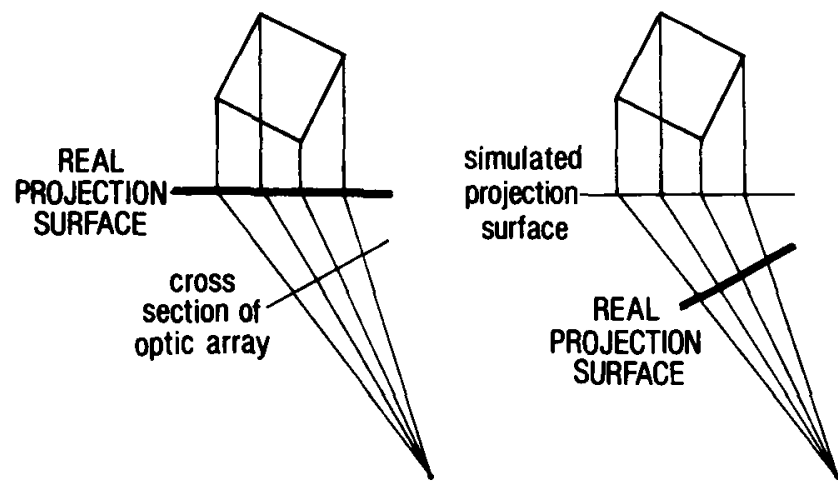

Figure 3. Arrangements of real and simulated projection surfaces. (The left panel shows front-row, side-aisle viewing of cinema; that is, the physical projection surface is slanted, and one can imagine another surface cutting across the optic array as it projects to the viewer. In the right panel, the two projection surfaces are logically reversed so that slanted cinema can be projected to a viewer without surface information about screen slant.)

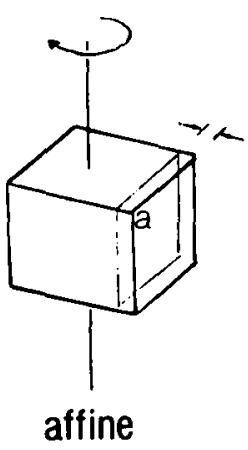

STIMULUS

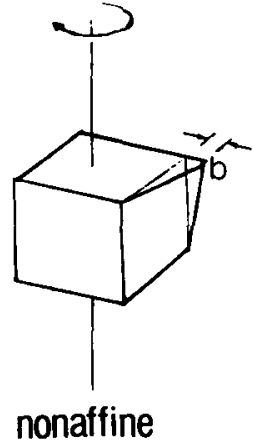

DEFORMATIONS
Figure 4. Two kinds of nonrigidities in the rotating solids judged by observers. (Because objects were deformed and rotated simultaneously during a stimulus trial, nonrigidity was not patent except when deformations were very large. The solids are shown in parallel projection. Corner $a$ is a "fork," and Corner $b$ an "arrow.")

(polar projection), and others appeared far away but enlarged as if through a telescope (parallel projection). In both cases the observer viewed the solid from above the plane of the top surface of the solid, as indicated in Figure 4. Second, cinematic viewpoint was varied through simulated slants of the projection screen. On many trials the optics of the stimuli seen were like those seen when sitting directly in front of the projection screen; in others they were more like those seen when sitting in the front row, side aisle of a movie theatre. For stimuli in these conditions, horizontal plane ( $x$ and $z$ ) dimensions of the solid were expanded so that when viewed from a simulated angle of less than $90^{\circ}$, they would have the same horizontal subtense as those viewed from a simulated $90^{\circ}$. Without such expansion, these objects would look substantially narrower than those viewed at $90^{\circ}$ and would perhaps clue the viewer to experimental manipulations. This expansion changed neither the process of reconstructive geometry nor the affine and perspective transformations of the solid. Third, many of the solids were computed to be rigid throughout rotation, and many were nonrigid.

Viewers were the author and 5 graduate students at Cornell University who were familiar with psychophysical procedures but unfamiliar with the hypotheses or intents of the experiments. The author and 2 others participated in all three experiments, but the latter viewers were not debriefed until after the third experiment. No viewers but the author had a priori information about the set of stimuli except that some would appear rigid, some would appear nonrigid, and others would be difficult to discern. Subjects sat in a moderately lit room (to diminish effects of phosphor persistence), with the face and edges of the actual display surface clearly visible. In each experiment, viewers made a scaled judgment of confidence and rigidity: 9 indicated high confidence that the object was rigid, 1 indicated high confidence that it was nonrigid, and 5 indicated lack of sureness.

\section{Experiment 1: Slanted Cinema Without Projection Surface Information}

Experiment 1 had two goals. First, it investigated the necessity of Euclidean rectification in the perception of dynamic

\footnotetext{
${ }^{7}$ Another reason that hidden lines were removed is that the rotating solids often spontaneously reversed and became rotating plastic objects. See Schwartz and Sperling (1983) for a similar phenomenon.
} 
slanted images. If rectification through surface slant, specularity shape, or binocular disparities is unnecessary, then objects projected to the observer through the double projection system at, say, $67^{\circ}$ rather than $90^{\circ}$ ought to be perceived as equally rigid. If such rectification is necessary, then such stimuli ought to be seen as less rigid.

Second, it investigated an aspect of the geometry that might be used by the visual system in perceiving rotating, rectangular solids. This was accomplished by presenting nonrigid as well as rigid stimuli to observers; the nonrigid stimuli underwent different kinds of rigidity violations. I call them nonrigid deformations. The two chosen were affine and nonaffine. If the visual system were more sensitive to one than to the other, implications could be drawn with respect to the indiscriminable distortion explanation of La Gournerie's paradox.

\section{Method}

The design had six factors of empirical interest. First, half of the stimuli were parallel projected (i.e., seen as if at infinite viewing distance but enlarged as if through an infinitely powerful telescope); half were polar projected at a viewing distance of 20 stimuli radii (which is not a high degree of perspective) and enlarged to the same degree. Second, half of the stimuli were projected through the double-projection system with surfaces parallel, simulating what 1 designate as a $90^{\circ}$ viewing angle, and half had an angle of $22,5^{\circ}$ between them and simulated a viewing angle of 67", as shown in Figure 3. Third, half of the stimuli were computed to be rigid, half nonrigid. Fourth, nonrigid stimuli underwent one of two kinds of geometric deformation during rotation: Half underwent an affine deformation, half nonaffine. As shown in Figure 4, affine deformations compressed and expanded the solid along one of its axes orthogonal to the axis of rotation. In the two-dimensional image, this deformation generally preserves the angles of intersections at the corners of the solid; preservation is exact across all frames for parallelprojected stimuli and nearly exact for polar-projected stimuli, depending on the relative distance of the observer from the solid. This deformation, however, does not preserve information about the shapes of sides across frames. In nonaffine deformations, on the other hand, one corner of the solid moved along these axes to the same degree. This deformation preserves neither the angles of intersection nor the shapes of sides (and in fact should make one surface look somewhat curved or hinged). The particular affine transformation was chosen because it was a global stimulus change without locally measurable alterations, whereas the nonaffine transformation created a definite local stimulus change. Both deformations had sinusoidal excursions and were accomplished during one complete rotation of the stimulus.

Fifth, the particular face (for affine deformations) or corner (for nonaffine deformations) involved in deformation was varied. For faces, any one of the four faces parallel to the vertical axis of rotation was moved during a given trial; for corners, any one of the four corners on one of the larger faces parallel to the vertical axis was moved along either $x$ or 2 axes of the solid. Sixth, the outward-then-inward excursion of a rigidityviolating face or corner could be $32 \%, 16 \%$, or $8 \%$ of the shortest radius of the solid. Large excursions of either kind would be expected to be relatively easy to see, making the solid appear nonrigid, and small ones would be much harder to see.

Six observers viewed 384 trials each: 2 perspectives (parallel and polar) $\times 2$ simulated viewpoints $\left(90^{\circ}\right.$ and $\left.67^{\circ}\right) \times 2$ types of stimuli (rigid and nonrigid) $\times 2$ types of nonrigidity (affine and nonaffine) $\times 3$ extents of rigidity violation $(32 \%, 16 \%$, and $8 \%) \times 4$ different faces or corners $\times$ 2 replications of each stimulus type. The fourth through seventh factors apply only to nonrigid stimuli, but there were equal numbers of rigid stimuli matched to nonrigid stimuli. Stimulus presentation was blocked
Table 1

Selected Mean Judgments From Experiment 1

\begin{tabular}{lll} 
& \multicolumn{2}{c}{$\begin{array}{c}\text { Simulated screen } \\
\text { slants }\end{array}$} \\
\cline { 2 - 3 } Stimulus class & $90^{\circ}$ & $67^{*}$ \\
\hline Simulated viewing distance & & \\
Infinite radii & 6.76 & 6.71 \\
20 radii & 6.25 & 6.40 \\
Rigid stimuli & 7.46 & 7.50 \\
Nonrigid stimuli & & \\
Affne deformed & 6.49 & 6.60 \\
Nonaffine deformed & 4.61 & 4.60 \\
Extent of nonrigid deformation & & \\
0.32 radii & 4.38 & 4.04 \\
0.16 radii & 5.49 & 5.68 \\
0.08 radii & 6.77 & 7.09 \\
\hline
\end{tabular}

Note. Judgments were made on a scale from 1 (for items that appeared least rigid) to 9 (for items that appeared most rigid). Standard errors of the mean (across observer means) average .90 per cell.

by perspective and by extent of rigidity violation. Because each stimulus was computed between trials (which took roughly 30 s), the entire experiment involved about $6 \mathrm{hr}$ of viewing per observer, distributed over about a month's time.

\section{Results and Discussion}

In presenting the results, let me proced through the various factors, discussing effects as I go. Basic results are given in Table 1, parsed according to the two simulated slants. First, and most surprising, there was no effect of simulated viewing angle, $F(1$, $5)=0.19, p=.68$; mean judgments were 6.50 for the $90^{\circ}$ double projections and 6.55 for those at $67^{\circ}$. Coupled with the fact that there were no interactions of viewing angle with other factors, these results strongly suggest that Euclidean rectification is not necessary for the perception of cinematic objects seen from the front row, side aisle. It may be either that useful perceptual information persists under noncomposition-point viewing conditions (the invariance hypothesis) or that the visual system is simply not attuned to certain screen slant transformations of dynamic stimuli (the indiscriminability hypothesis).

Second, there was no reliable difference between parallel- (infinite radii) and polar- (20 radii) projected stimuli, $F(1,5)=$ $1.73, p=.245$. Mean judgments of rigidity were 6.73 and 6.32 , respectively. The direction of this difference in judgments is consistent with the literature, as discussed below. The lack of an effect, as will be borne out by later experiments, is due to the relatively low perspective in the polar stimuli.

Third, as might be expected, there was a reliable effect of rigid versus nonrigid stimuli, $F(1,5)=21.98, p<.005$. More important, among nonrigid stimuli, there was a substantial effect of type of nonrigidity, $F(1,5)=10.66, p<.02$; all affine deformed stimuli received mean judgments of 6.54 , and all non-

\footnotetext{
This affine deformation is only one of a class of such deformations that might be used. Others would make rectangular solids into parallelepipeds.
} 
affine ones received 4.61. This result demonstrates that ongoing affine deformations of rotating stimuli are less well discerned by the perceiver. Moreover, because the affine deformation preserved all angular intersections at the corners of the rotating solid, whereas the nonaffine deformation did not, this result implicates local comparisons and local tracking of line intersections across time as the information used in making rigidity judgments.

Finally, also as expected, there was a sizable effect of the extent of nonrigid excursion of a face or corner, $F(2,10)=33.21$, $p<.001$, with judgments of $4.20,5.59$, and 6.93 for excursions of $32 \%, 16 \%$, and $8 \%$ of the stimulus radius, respectively.

Static versus motion information. Whereas many of the stimuli were generally seen as rigid and many as nonrigid, it is not clear from the data just presented whether the information used by observers was static or whether it accrued over time in the motion of the rotating solids. Because static information can dominate motion information (Schwartz \& Sperling, 1983), it would be useful to try to determine which was used by observers here.

One possible source of static information concerns the special character of these stimuli. All possible projections of rectangular objects, according to Perkins (1968), should follow two constraints. Kubovy (1986) called these Perkins's laws. Perkins's first law concerns three-way intersections of edges that are interior (not at the perimeter) in the image. These are known as "forks," and one is labeled $a$ in Figure 4. To be a proper corner of a rectangular solid, the projection of each of the three right angles of that corner must be greater than $90^{\circ}$. Perkins's second law concerns three-way intersections of corners on the perimeter of the object's image. These are known as "arrows," and one is labeled $b$ in Figure 4 . The two right angles projected in each arrow must be less than $90^{\circ}$, and the sum of the two must be greater than $90^{\circ}$.

The logic of application of these laws (which were devised for static figures) to rotating, rectangular solids is as follows: If a particular corner violates a law in some frames of the stimulus sequence but not others, then it is possible that the object might be seen as nonrigid, at least near that corner. No frame of any stimulus sequence in this experiment or in the following two experiments violated Perkins's (1968) first law. However, there were some stimuli in Experiment 1 and in Experiments 2 and 3 that violated the second law. In all cases it was either the upper right corner ( $b$ in Figure 4 ) or the lower right corner, and in all cases the sum of the two projected angles was less than $90^{\circ}$.

When stimuli were collapsed across replications and face/ corner manipulations, there remained 48 different stimuli in Experiment 1. For these, the correlation between number of violations across frames within a stimulus sequence and perceived rigidity was quite high $(r=-.80), t(46)=9.04, p<.001$; that is, the more frames with violations there were, the less rigid the object appeared. Thus, by an argument based on Perkins's (1968) laws, there is evidence that perceivers used static information to make their judgments.

Overview. These results argue three things for the perception of cinema seen from the front row, side aisle. First, they negate the necessity of Euclidean rectification as an explanation of La Gournerie's paradox in cinema, at least through the use of frame distortions, surface information, and binocular dispari- ties of screen slant. Second, stimulus information about rotating solids appears to survive certain noncomposition-point viewing, and observers appear to use this information. Third, perception of nonrigidities in these stimuli could be based on static information, particularly the projected relations among intersecting edges.

The first conclusion is the most important, and against it there are two arguments. First, it might be that screen slant is derived not from surface information but from optical information in the stimulus across frames. Second, one could argue that in $67^{\circ}$ viewing, one does not use a sufficiently slanted screen; distortion effects could be much more easily noted when the cinema screen is at a more acute angle. In Experiment 2, I considered both of these issues.

\section{Experiment 2: Constant and Variable Screen Slants Without Projection Surface Information}

Experiment 2 had four goals. The first was to explore the possibility that screen slant could be computed across frames from stimulus optics, rendering rectification possible, perhaps likely, in slanted cinema viewing. The second was to explore a more extreme slant. These goals were met by using four viewing conditions: simulated slants of $90^{\circ}$ and $67^{\circ}$, as in Experiment 1, and two others. To meet the second goal, a constant screen slant of $45^{\circ}$ was added; to meet the first, a variable screen slant condition was included (discussed below).

The third goal was to explore a different range of simulated viewing conditions. Experiment 1 considered true parallel projections and at polar projections of 20 stimulus radii; this experiment considered 2 closer to the stimuli. The expectation was that either a main effect of or an interaction with viewing distance would accrue. The fourth goal was interwoven with the third, and it was to compare the efficacy of the simulated viewing distances with physical viewing distances; that is, viewers were moved physically closer or farther from the screen at distances that would either match or mismatch the simulated viewing distances. In such manner the effect of projected optics versus screen optics can be separated, and the role of surface information on the display scope can be better assessed.

\section{Method}

This experiment also had six experimental factors. The first, as before, was parallel versus polar projection. Simulated viewing distances were 30 stimulus radii (approximating parallel projection) and $10 \mathrm{stim}$ ulus radii. Second, however, physical viewing distance from the screen was varied in such a way that the observer was actually 30 and 10 stimulus radii from the computed location of the object. Because these two factors were crossed, the observer could be at, closer than, or farther than the composition point for all $90^{\circ}$ viewing positions.

Third, there were four simulated viewing slants: constant $90^{\circ}, 67^{\circ}$, and $45^{\circ}$, and a variable screen slant condition that averaged $67^{\circ}$. In the latter condition the angle between the simulated and real projections surfaces oscillated sinusoidally, yielding slants that varied between $80^{\circ}$ and $55^{\circ}$ twice during each stimulus rotation, six times during a trial. As before, a constant compensation for the horizontal dimensions of the solid were made for stimuli in all slanted conditions. This means that in the variable-slant condition, the projected width of the object varied within a stimulus sequence as much as $8.5 \%$ in either direction from its expected value. Static frames taken out of a dynamic sequence are 
shown in Figure 5 for $90^{\circ}, 67^{\circ}$, and $45^{\circ}$ screen-slant conditions for nearparallel and polar projections.

The other three factors were as before: Stimuli were rigid and nonrigid; nonrigid stimuli had affine and nonafine deformations; and surfaces (for aftine deformations) and comers (for nonaffine deformations) invol ved in rigidity violation were varied. Extent of the nonrigid excursions was kept constant at $16 \%$. Unlike the stimuli in Experiment 1 , only one ninth of all stimuli were rigid, and eight ninths were nonrigid in this study. Otherwise, conditions were identical.

Three participants viewed 576 stimulus sequences-64 that were rigid, 512 nonrigid. Among nonrigid trials the factors were 2 simulated viewing distances ( 30 and 10 stimulus radii) $\times 2$ physical viewing distances ( 30 and 10 stimulus radii) $\times 4$ simulated viewing slants $\left(90^{\circ}, 67^{\circ}\right.$, and $45^{\circ}$, and variable $67^{\circ}$ ) $\times 2$ types of nonrigidity (affine and nonaffine) $\times 4$ faces or corners $\times 4$ replications per stimulus type. Rigid stimuli had all factors except type of nonrigidity and face/corner variation. Because all stimuli were precomputed for this experiment, total viewing time for each observer was about $2 \mathrm{hr}$, distributed over a week.

\section{Results and Discussion}

Because of the unequal number of rigid and nonrigid trials, these were separated in all analyses. Moreover, because there were no reliable effects across rigid trials, all those reported are for nonrigid stimuli. Also, given that only 3 observers participated but that each viewed many trials, all analyses (except correlations) are reported for individuals rather than the group. Major results are given in Table 2, parsed according to the four simulated screen slant conditions.

First, there was a substantial effect for simulated viewing angle for all 3 observers, all $F \mathrm{~s}(3,9)>7.39$, all $p \mathrm{~s}<.008$. However, mean judgments across observers revealed a pattern not anticipated. Surprisingly, the main effect was due to low judgments of (increased nonrigidity seen in) the $45^{\circ}$ stimuli; there were no reliable differences among the other three conditions. This result answers two questions about Experiment 1. First, as one would have expected, distortions in stimuli presented on slanted screens are detectable when the slant is great enough. Such a result is in keeping with the analyses of Meister (1966), who calculated distortions in cineauditoriums but gathered no empirical data. Second, because the rigidity judgments of stimuli in the constant $90^{\circ}$ and $67^{\circ}$ conditions and in the variable $67^{\circ}$ condition were the same and because it would be dificult to compute slant in any single frame or to calculate sinusoidal screen movement during stimulus presentation, the computation and use of screen slant seems unlikely during perception of these objects.

Second, there was a main effect of simulated viewing distance from the rotating object for all 3 observers, all $F s(1,3)>10.99$, ps $<.045$; stimuli at 30 radii received mean judgments of 5.63 , and those at 10 radii received 4.74 . This type of effect is often found in studies varying presentations of stimuli between parallel and polar projections (Braunstein, 1976; Hagen \& Elliott, 1976; Hagen, Elliott, \& Jones, 1978). Hagen and her colleagues called it the zoom effect, in which the preferred optics of pictorial perception appear to be those of seeing through a telephoto lens, which provides a more parallel projection than would be the case in normal viewing. In my study, however, this zoom effect means that nonrigidity is harder to detect in parallel-projected displays, not that they are preferred.
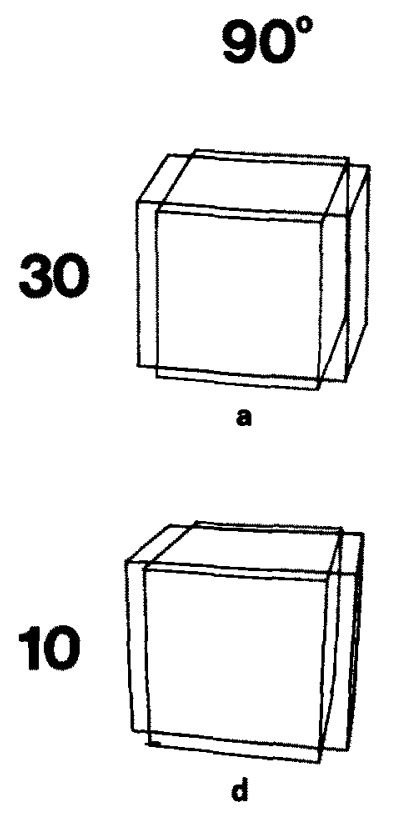
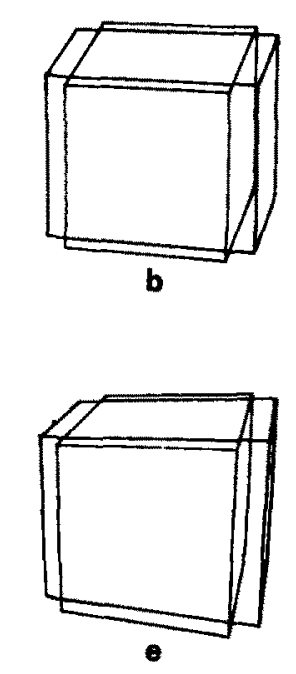

b
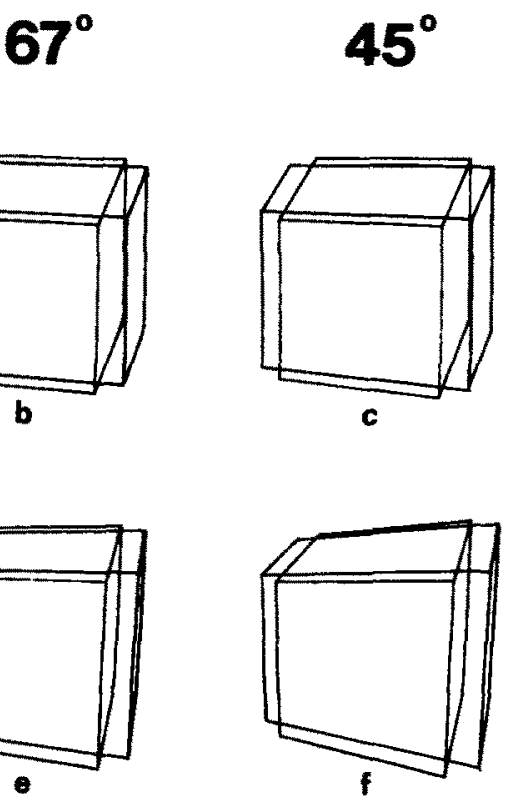

Figure 5. Static frames taken out of a dynamic sequence for solids seen at simulated slants of $90^{\circ}, 67^{*}$, and 45* for near-parallel ( 30 radii) and polar ( 10 radii) stimuli. (Stimuli in the slanted frames are expanded in their $x z$ planes so that their horizontal subtense is the same as the unslanted stimuli. Frames superimposed in each panel are those for a stimulus rotation of $90^{\circ}$. Stimuli in Panels $\mathrm{c}$, $e$, and $\mathrm{f}$ violate Perkins's (1968) second law in their upper right comers; the stimulus in Panel $f$ also violates that law in the lower right corner.) 
Tabie 2

Selected Mean Judgments From Experiment 2

\begin{tabular}{lllll}
\hline & \multicolumn{4}{c}{ Simulated screen slant } \\
\cline { 2 - 5 } \multicolumn{1}{c}{ Stimulus class } & $90^{\circ}$ & $67^{\circ}$ & Variable $67^{\circ}$ & $45^{\circ}$ \\
\hline Simulated viewing distance & & & & \\
$\quad 30$ radii & 5.61 & 5.78 & 5.73 & 5.40 \\
$\quad$ 10 radii & 5.62 & 5.03 & 5.19 & 3.10 \\
Physical viewing distance" & & & & \\
30 radii & 5.45 & 5.24 & 5.31 & 4.04 \\
10 radii & 5.78 & 5.57 & 5.61 & 4.46 \\
Rigid stimuli & 8.02 & 7.21 & 7.25 & 5.83 \\
Nonrigid stimuli & & & & \\
$\quad$ Affine deformed & 7.66 & 7.34 & 7.53 & 5.83 \\
$\quad$ Nonaffine deformed & 3.57 & 3.43 & 3.40 & 3.00 \\
\hline
\end{tabular}

Note. Judgments were made on a scale from 1 (for items that appeared least rigid) to 9 (for items that appeared most rigid). Standard errors of the mean (within individual observers, then averaged across them) across cells were .85 for the rigid stimuli and .42 for other cells.

These means are for nonrigid trials only.

Third, there was a reliable effect of physical viewing distance from the screen for only 1 observer, $F(1,3)=77.9, p<.01$; stimuli actually viewed at 30 radii garnered mean judgments of 5.01 across all observers, and those actually viewed at 10 radii received 5.36, an effect that was, if anything, the reverse of that for simulated distance. The two effects of simulated and physical viewing distance were without interaction.

Together, these second and third results appear to implicate the optics measured on the screen as being more important than those measured at the eye, which supports a Euclidean rectification view. But one must remember that these data are collapsed across the various screen-slant conditions. When only the $90^{\circ}$ viewing angle condition is included in analysis, no observers demonstrated an effect of simulated viewing distance, only one showed an effect of physical viewing distance, and no one showed an interaction. I take the principal result here to be that simulated and physical distances did not affect judgments for $90^{\circ}$ stimuli.

Fourth, among nonrigid stimuli, nonaffine-deformed objects were again seen as considerably less rigid than were affine-deformed stimuli; average judgments of these objects were 3.35 and 7.02, respectively, all $F \mathrm{~s}(1,3)>70.9$, ps $<.004$. In fact, because rigid stimuli received judgments of 7.07 , there was no perceived difference between rigid and affine-deformed nonrigid stimuli. Unlike Experiment 1, the difference between judgments of the two types of nonrigidity in this study was not attributable solely to static information, at least as defined by the number of violations of Perkins's (1968) second law within stimulus sequences. Although the correlation between number of frames in violation and mean stimulus judgments was reliable $(r=-.39), t(61)=9.7, p<.001$, it was well below the point-biserial correlation between type of nonrigidity and judgments $(r=.90), t(61)=25.5, p<.001$. This later correlation can be taken as the motion (as opposed to static) information about nonrigidity in the stimuli. Together the two variables accounted for $92 \%$ of the variance in the nonrigid stimulus judgments.

Last, one interaction is of interest, reliable across all 3 observ- ers and suggested by the results just presented. This was the relation between simulated projection distance ( 30 vs. 10 radii) and simulated screen-slant conditions $\left(90^{\circ}, 67^{\circ}, 45^{\circ}\right.$, and variable $\left.67^{\circ}\right)$, all $F \mathrm{~s}(3,9)>8.7, p \mathrm{~s}<.001$. This effect was due to the fact that polar stimuli (10 radii) viewed at $45^{\circ}$ were seen as less rigid (given mean judgments of 3.10 ) than the stimuli of all seven other conditions (5.48). In particular, stimuli projected from a greater distance ( 30 radii) but with equal slant received much higher judgments (5.40). This effect, collapsed across relevant stimuli, seems well explained by the number of violations of Perkins's (1968) second law within a stimulus sequence $(r=$ $.96), t(6)=30.0, p<.001$. This interaction lends yet another kind of substance to the zoom effect than originally intended. Hagen and Elliott (1976; Hagen et al., 1978) suggested that parallel projections in pictures were preferred to polar projections because they are more viewpoint independent; in this study parallel projected stimuli survived distortions of slanted screens better and thus serve La Gournerie's paradox. Said another way, there is no paradox for polar projected objects on highly slanted screens; these stimuli actually look bad.

\section{Overview}

Four results are of theoretical importance. First, and I think most important, variable screen slant did not affect judgments of rigidity. This result, I claim, argues against the use of a constant screen slant variable in perception because it would have to be continually updated in order to interpret the rotating solid as rigid. Such computation seems implausible, given the unknown and uncorrelated motion of the screen. Second, conditions of simulated screen slants of as much as $45^{\circ}$ do show noticeable distortions and create many violations of Perkins's (1968) second law. This point of perceptual breakdown is close to, but perhaps occurs earlier than, those at which perception of objects with real screen slants may break down (Meister, 1966; Perkins, 1972, 1973; but see Gibson, 1947). Third, these distortions are particularly noticeable only for polar-projected stimuli, again because of violations of Perkins's second law. Thus La Gournerie's paradox occurs differentially and only for more parallel projections. Fourth, however, motion information alone (as reflected in the difference in judgments between affineand nonaffine-deformed stimuli) can be very important in revealing nonrigidity.

\section{Experiment 3: Anamorphic Cinema}

Experiment 3 had one general goal-to explore the role of the optics of projection on the screen and projected to the eye in terms of simulated and physical projection distances and simulated and real screen slants. Within this goal, however, was a more particular subgoal: Because the previous experiments demonstrated the nonnecessity of Euclidean rectification as a solution to La Gournerie's paradox in cinema, here I explored its potential nonsufficiency; that is, if an observer looks at a physically slanted screen but sees an object whose optics are those of a nonslanted stimulus, as in anamorphic art (see Leeman, Elffers, \& Schuyt, 1976, and Battisti, Carnemolla, Masters, \& Menna, 1981), then Euclidean rectification would actually hinder perception of rigidity. 


\section{Method}

Five factors are of empirical interest. As in Experiment 2, two dealt with projected distance of the stimuli, simulated and real. Both entailed viewing distances of $37.5,15$, and 6 stimulus radii. Two other factors dealt with screen slant: $90^{\circ}, 67^{\circ}$, and $45^{\circ}$, again both simulated and real, but in opposite directions. Simulated slants, as before, were clockwise (seen from above), as if one were seated in a theater down front and to the right; real slants were counterclockwise. As in Experiments 1 and 2, the simulated screen slant was through the double projection system, but unlike in previous experiments, the real screen could be slanted as well. The intent of this manipulation was, for certain conditions, to cancel simulated and real-screen slants and to create anamorphic cinema. The fifth factor was rigidity: Half of the stimuli were rigid and half nonrigid, and all nonrigid stimuli underwent affine deformation.

Three observers participated in 648 trials each: 3 simulated projection distances $\times 3$ physical projection distances $\times 3$ simulated screen slants $\times 3$ real screen slants $\times 2$ stimulus states (rigid and nonrigid) $\times 4$ replications of each stimulus type. Again, because stimuli were precomputed, total viewing time per viewer was about $2 \mathrm{hr}$, distributed over a week.

\section{Results and Discussion}

Again, results are discussed by factor. Again, initial analyses were performed on individual data, but this time they included all trials, rigid and nonrigid. First, only 2 of the 3 observers demonstrated reliable effects for rigid versus nonrigid stimuli; means across observers were 6.92 for rigid stimuli and 6.44 for the affine-deformed nonrigid stimuli.

Second, as before, there were no consistent and reliable effects of physical distance from the projection screen; mean judgments were $6.72,6.85$, and 6.48 for distances of $37.5,15$, and 6 stimuli radii, respectively. But all 3 observers showed reliable main effects for simulated distance from the rotating object, all $F s(2,6)>29.9, p s<.001$; mean judgments were 7.69 , 7.21 , and 5.15 for the same three distances, respectively. This effect, however, was due in interaction to the various screen slants, as discussed below; there were no consistent effects of simulated distance (for example, for $90^{\circ}$ stimuli). Thus I take the main result of the distance analyses here, as in Experiment 2 and contrary to the general literature (Braunstein, 1976), to be that neither physical nor simulated distances affected rigidity judgments of stimuli seen on unslanted screens.

Third, there were no consistent and reliable effects of real screen slant; mean judgments were $6.66,6.73$, and 6.66 , respectively, for $90^{\circ}, 67^{\circ}$, and $45^{\circ}$ tilts of the actual display monitor in front of the viewer. There were, however, consistent and reliable effects of simulated screen slant, all $F \mathbf{S}(2,6)>74.2, p s<.001$; mean judgments were $7.43,7.07$, and 5.54 , respectively, for the three slants. Again, it appears that these results support Euclidean rectification, especially because of the difference in the two $45^{\circ}$ conditions. But the reason for the difference between real and simulated slants is more complex. Consider a result that introduces this idea.

One interaction was consistent across observers. Simulated viewing distance and simulated projection slant, as in Experiment 2 , interacted reliably, all $F \mathrm{~s}(4,12)>14.78$, ps $<.001$. This result is best seen in Table 3 . There was, for example, no difference among judgments for near-parallel projected stimuli (37.5 radii) at various slants, but there was a substantial differ-
Table 3

Selected Mean Judgments from Experiment 3

\begin{tabular}{cccc}
\hline & \multicolumn{3}{c}{ Simulated screen slant } \\
\cline { 2 - 4 } Simulated viewing distance & $90^{\circ}$ & $67^{\circ}$ & $45^{\circ}$ \\
\hline 37.5 radii & 7.56 & 7.78 & 7.72 \\
15 radii & 7.80 & 7.93 & 5.89 \\
6 radii & 6.93 & 5.50 & 3.03 \\
\hline
\end{tabular}

Note. Judgments were made on a scale from 1 (for items that appeared least rigid) to 9 (for items that appeared most rigid). Standard errors of the mean (within individuals, then averaged across individuals) across cells were .14; they are smaller in this table because there were no nonaffine-deformed stimuli.

ence for high-perspective (6 radii) stimuli. In other words, as before, low-perspective (near-parallel) projections look reasonably rigid from the side, and high-perspective projections look very nonrigid. This pattern of results fits quite snugly with an analysis of the number of stimulus frames per sequence in which Perkins's (1968) second law is violated $(r=-.96), t(7)=$ $32.5, p<.001$.

The final and more selective analyses are most important to my argument concerning La Gournerie's paradox. They concern the nonsufficiency of Euclidean rectification. For the nearparallel projected stimuli (37.5 radii), the perspective distortions of complementary simulated and real screen slants of $67^{\circ}$ and $45^{\circ}$ effectively cancel for the farthest physical viewing distance: ${ }^{9}$ The optics presented to the observer through these double slants are like anamorphic film. Thus the stimulus sequences made for side viewing have optics like those of head-on viewing.

If one considers real and simulated slants of $90^{\circ}$, which I call $90 / 90$, and compares them with siants of $67 / 67$ and $45 / 45$ (where the first number is the physical slant and the second the simulated slant in the opposite direction), then there were no reliable differences for any of the observers. Means of these three conditions were $7.91,7.88$, and 7.63 , respectively, $F \mathrm{~s}(1$, $2)=1.32,1.86$, and 3.25 for the 3 observers (all $p s>.10$ ). Thus rigidity seen in these anamorphic sequences is not reliably more difficult than those with unslanted screens. Moreover, according to a Euclidean rectification account, judgments of rigidity would be greater in regular slanted cinema conditions (67/90 and $45 / 90$ in my terminology) than in anamorphic conditions $(67 / 67$ and 45/45). In fact, if anything, the reverse was true: There was a mean judgment of 7.75 across the $67 / 67$ and $45 /$ 45 conditions and a mean of 7.40 across the $67 / 90$ and $45 / 90$ conditions, $F(1,2)=10.98, p=.08$, across all observers. Clearly, even though the physical surface slant of the screen could be seen in Experiment 3, there is no evidence for its use in making rigidity judgments.

\footnotetext{
${ }^{9}$ Optical cancellations of the two screen slants, simulated and real, can occur only for parallel and near-parallel projections viewed at some distance. Distortions of the left and right edges of the solid, for example, are within the resolution error of my display device and within $0.5 \mathrm{~min}$ of are visual angle. However, the cancellations occur only for the perspective distortions; objects seen through cancelling screen stants will always appear narrower than those with unslanted screens.
} 


\section{Conclusions}

La Gournerie's paradox concerns the unnoticed distortions in the optics of objects in a photograph or cinema, particularly when viewed from the side. The fact that these distortions are unnoticed is fortunate, but it is a puzzle for linear perspective and is unpredicted by the rigorous rules of projections. In fact, these projective rules dictate that such objects rotating in a film should appear nonrigid, yet they rarely are.

Three classes of explanations for this paradox were broached, two discussed in detail. The first, what I call Euclidean rectification, is a procedure in which surface cues of the slanted screen itself (or stimulus optics) are used to rectify the relations of virtual space. My data do not support this view. In particular, such rectification is not necessary because judgments of objects on slanted screens without cues to slant (and on even screens that oscillate) are no different than those for objects seen head on. Moreover, such rectification is not sufficient to explain the results of anamorphic cinema, in which objects appear no less rigid (and perhaps even more rigid) than those on simple slanted screens. ${ }^{10}$

A second explanation concerns the invariance hypothesis. This view was untested. Its problem, at least from my view, is that mathematical invariants tend to be highly specific, particularly to forms. The cross ratio, for example, cannot be generalized beyond coplanar parallel lines. It seems unlikely that different geometric solids have a general invariant or that each possible solid has a specific one. ${ }^{11}$

The third class of explanation, which I favor in this context and which is generally supported by my data, is that local distortions in moderately slanted cinema are sufficiently small as to be unregistered by the visual system. Said differently, information for making certain judgments about certain objects, such as rigidity in rectangular solids, is sufficiently well preserved in the optics of slanted screens so that perception is unperturbed. Analysis of the angular intersections of edges of the rectangular solids, known as Perkins's (1968) laws, demonstrate that the rigidity judgments appear to be based on local angular measurements. These, of course, cannot generally hold because most cinematic objects are without right-angled corners. What the information might be more generally is not clear. I suspect, simply on the basis of my own introspections and on Meister's (1966) data, that the tolerances when viewing slanted cinema are about the same for both rectangular and nonrectangular moving objects.

In summary, my claim is twofold: Film and television can be viewed from positions other than the composition point precisely because (a) the optics of parallel and near-parallel projections of objects are sufficiently robust against moderate screen slants seen from moderate distances, and (b) the human visual system is sufficiently inexact in its local measurements of optical projections to tolerate small distortions. This means that when one is observing the motions of a near-parallel projected object, a screen with moderate slant (say, about $67^{\circ}$ ) is functionally and psychologically no different than one without slant. Thus, at least for cinema, La Gournerie's paradox may be explained without reference to screen slant but with regard to optical distortions that are indiscriminably different from a veridical optical projection.

\begin{abstract}
${ }^{10}$ One might claim that rigidity is an invariant. I claim (Cutting, $1986 \mathrm{a}$, chap. 5), however, that rigidity is a property that may arise out of an invariant. I reserve the term invariant for mathematical statements about relations among optical measurements, expressed in terms of real numbers or ordered relations among reals.

"In faimess, it may be that Euclidean rectification is responsible for the perceptions of slanted pictures, but not for cinema. Although this view may be unparsimonious, pictures (particularly standard photographs) are viewed at close range, where cues of binocular disparity are very strong; cinema is often viewed (as in some conditions of this experiment) at a much greater distance.
\end{abstract}

\section{References}

Anstis, S. M., Mayhew, J. W., \& Morley, T. (1969). The perception of where a face or "portrait" is looking. American Journal of Psychology, $82,477-489$.

Barnard, S. T. (1985). Choosing a basis for perceptual space. Computer Vision, Graphics, and Image Processing, 29, 87-99.

Battisti, E., Carnemolla, A., Masters, R. J., \& Menna, F. (1981). Anamorphosis: Evasion and return. Rome: Officina Edizioni.

Bennett, B. M., \& Hoffman, D. D. (1985). The computation of structure from field-axis motion: Nonrigid structure. Biological Cybernetics, 51, 293-300.

Braunstein, M. L. (1976). Depth perception through motion. New York: Academic Press.

Braunstein, M. L., \& Payne, J. W. (1969). Perspective and form ratio as determinants of relative slant judgments. Journal of Experimental Psychology, 81, 584-590.

Cutting, J. E. (1986a). Perception with an eye for motion. Cambridge, MA: MIT Press/Bradford Books.

Cutting, J. E. (1986b). The shape and psychophysics of cinematic space. Behavior Research Methods, Instruments, and Computers, 18, 551558.

Epstein, W., Bontrager, H., \& Park, J. (1962). The induction of nonveridical slant and the perception of shape. Journal of Experimental Psychology, 63, 472-479.

Farber, J., \& Rosinski, R. R. (1978). Geometric transformations of pictured space. Perception, 7, 269-282.

Frangenberg, T. (1986). The image and the moving eye: Jean Pélerin (Viator) to Guidobaldo del Monte. Journal of the Warburg and Courtauld Institutes, 49, 150-171.

Gibson, J. J. (Ed.). (1947). Motion picture testing and research (Aviation Psychology Research Reports, No. 7). Washington, DC: U.S. Government Printing Office.

Gibson, J. J. (1979). The ecological approach to visual perception. Boston: Houghton Mifflin.

Greene, R. (1983). Determining the preferred viewpoint in linear perspective. Leonardo, 16, 97-102.

Guzman, A. (1969). Decomposition of a visual scene into three-dimensional bodies. In A. Graselli (Ed.), Automatic interpretation and classification of images (pp. 243-276). New York: Academic Press.

Hagen, M. A., \& Elliott, H. B. (1976). An investigation of the relationship between viewing condition and preference for true and modified linear perspective with adults. Journal of Experimental Psychology: Human Perception and Performance, 4, 479-490.

Hagen, M. A., Elliott, H. B., \& Jones, R. K. (1978). A distinctive characteristic of pictorial perception: The zoom effect. Perception. 7, 625633.

Hochberg, J. (1978a). Art and perception. In E. C. Carterette \& M. P. Friedman (Eds.), Handbook of perception (Vol. 10, pp. 225-256). New York: Academic Press.

Hochberg, J. (1978b). Perception (2nd ed.). Englewood Cliffs, NJ: Prentice-Hall. 
Hochberg, J., \& Brooks, V. (1978). The perception of motion pictures. In E. Carterette \& M. P. Friedman (Eds.), Handbook of perception (Vol. 10, pp. 257-304). New York: Academic Press.

Kubovy, M. (1986). The psychology of perspective and Renaissance art. London: Cambridge University Press.

Leman, F., Elffers, J., \& Schuyt, M. (1976). Hidden images. New York: Harry N. Abrams.

Lumsden, E. A. (1980). Problems of magnification and minification: An explanation of the distortions of distance, slant, shape, and velocity. In M. A. Hagen (Ed.), The perception of pictures (Vol. 1, pp. 91135). New York: Academic Press.

Meister, R. (1966). The iso-deformation of images and the criterion for delimitation of the usable areas in cinc-auditoriums. Joumal of the Society for Motion Picture and Television Engineers, 75, 179-182.

Olson, R. K. (1974). Slant judgments from static and rotating trapezoids correspond to rules of projective geometry. Perception \& Psychophysics, 15, 509-516.

Perkins, D. N. (1968). Cubic comers. Quarterly Progress Report of the MIT Research Laboratory of Electronics, 89, 207-214.

Perkins, D. N. (1972). Visual discrimination between rectangular and nonrectangular parallelopipeds. Perception \& Psychophysics, 12, $396-400$.

Perkins, D. N. (1973). Compensating for distortion in viewing pictures obliquely. Perception \& Psychophysics, 14, 13-18.

Perkins, D. N. (1982). The perceiver and organizer and geometer. In J. Beck (Ed.), Organization and representation in perception (pp. 7393). Hillsdale, NJ: Erlbaum.

Perrone, J. A. (1984). Visual slant misperception and the "black-hole" landing situation. Aviation, Space, Environmental Medicine, 55, 1020-1025.

Pirenne, M. H. (1970). Optics, painting, \& photography. Cambridge, England: Cambridge University Press.
Rock, I. (1983). The logic of perception. Cambridge, MA: MIT Press/ Bradford Books.

Rosinski, R. R., \& Farber, J. (1980). Compensation for viewing point in the perception of picture space. In M. A. Hagen (Ed.), The perception of pictures (Vol, 1, pp. 137-176). New York: Academic Press.

Rosinski, R. R., Mulholland, T., Degeiman, D., \& Farber, J. (1980). Picture perception: An analysis of visual compensation. Perception \& Psychophysics, 28, 521-526.

Schwartz, B., \& Sperling, G. (1983). Luminance controls the perceived 3-D structure of dynamic 2-D displays. Bulletin of the Psychonomic Society, 21, 456-458.

Shepard, R. N. (1981). Psychophysical complementarity. In M. Kubovy \& J. R. Pomerantz (Eds.), Perceptual organization (pp. 279-341). Hillsdale, NJ: Erlbaum.

Shepard, R. N., \& Cooper, L. A. (1982). Mental images and their transformation. Cambridge, MA: MIT Press/Bradford Books.

Ullman, S. (1979). The interpretation of visual motion. Cambridge, MA: MIT Press.

Ullman, S. (1983). Recent computational studies in the interpretation of structure from motion. In J. Beck, B. Hope, \& A. Rosenfeld (Eds.), Human and machine vision (pp. 459-480). New York: Academic Press.

Ulman, S. (1984). Maximizing rigidity: The incremental recovery of 3D structure from rigid and nonrigid motion. Perception, 13, 255274.

Wallach, $H$. (1976). The apparent rotation of pictorial scenes. In M. Henle (Ed.), Vision and artifact (pp. 65-69). New York: Springer.

White, J. (1957). The birth and rebirth of pictorial space. London: Faber and Faber.

Received October 26, 1986

Revision received December 17, 1986

Accepted January 18, 1987

Correction to Kay et al.

In the article "Space-Time Behavior of Single and Bimanual Rhythmical Movements: Data and Limit Cycle Model" by B. A. Kay, J. A. S. Kelso, E. L. Saltzman, and G. Schöner (Journal of Experimental Psychology: Human Perception and Performance, 1987, Vol. 13, No. 2, pp. 178-192), there was an error in Equation A3 of Appendix A. That equation should read:

$$
\dot{B}=-\frac{\alpha B}{2}-\frac{\gamma|B|^{2} B}{2} \text {. }
$$

